\title{
The relevance of non-stationarities and non-Gaussianities in vibration fatigue
}

\author{
Martin Česnik ${ }^{1}$, Janko Slavič ${ }^{1, *}$, Lorenzo Capponi ${ }^{2}$, Massimiliano Palmieri ${ }^{2}$, Filippo Cianetti ${ }^{2}$, and Miha Boltežar $^{1}$ \\ ${ }^{1}$ University of Ljubljana, Faculty of Mechanical Engineering, 1000 Ljubljana, Slovenia \\ ${ }^{2}$ University of Perugia, Department of Engineering, 06125 Perugia, Italy
}

\begin{abstract}
In classical fatigue of materials, the frequency contents of dynamic loading are well below the natural frequencies of the observed structure or test specimen. However, when dealing with vibration fatigue the frequency contents of dynamic loading and structure's dynamic response overlap, resulting in amplified stress loads of the structure. For such cases, frequency counting methods are especially convenient. Gaussianity and stationarity assumptions are applied in frequency-domain methods for obtaining dynamic structure's response and frequency-domain methods for calculating damage accumulation rate. Since it is common in real environments for the structure to be excited with nonGaussian and non-stationary loads, this study addresses the effects of such dynamic excitation to experimental time-to-failure of a structure.

Initially, the influence of non-Gaussian stationary excitation is experimentally studied via excitation signals with equal power density spectrum and different values of kurtosis. Since no relevant changes of structure's time-to-failure were observed, the study focused on non-stationary excitation signals that are also inherently non-Gaussian. The non-stationarity of excitation was achieved by amplitude modulation and significantly shorter times-to-failure were observed when compared to experiments with stationary non-Gaussian excitation.

Additionally, the structure's time-to-failure varied with the rate of the amplitude modulation. To oversee this phenomenon the presented study proposes a non-stationarity index which can be obtained from the excitation time history. The non-stationarity index was experimentally confirmed as a reliable estimator for severity of non-stationary excitation. The non-stationarity index is used to determine if the frequencydomain methods can safely be applied for time-to-failure calculation.
\end{abstract}

\section{Introduction}

Before it's exploitation a product (structure) must undergo a number of tests to prove its durability when exposed to harsh environmental conditions (vibrations, temperature, humidity, etc.). When discussing product's resistance to vibrations an automotive, aerospace and other testing standards [1] specify vibration loads that should be applied to a product in a controlled laboratory conditions via electro-magnetic shaker. Potential failure of product during vibration testing mainly occurs due to wear or fatigue fracture. In such cases of fatigue failure, the damage accumulation is not directly correlated to vibration load, but is rather coupled in frequency domain with structure's dynamic properties [2].

Thus, when dealing with vibration fatigue of structure under random vibration loads the counting methods in frequency domain (Dirlik [3], TovoBenasciutti [4]) are significantly more applicable, computationally effective and faster [5] compared to counting methods in time domain (rainflow, range count), especially when long time-histories need to be evaluated. Fatigue life estimation with frequency counting method is based on power spectral density (PSD) function of structure's stress response and properties of multiaxial stress criterion $[6,7]$. Inherently to this, a frequency counting methods assume signal's stationarity [8]. Furthermore, due to reasons of analytical deduction, an additional assumption of Gaussian nature of stress signal should be sufficed. When both assumptions are respected, the frequency methods give reliable fatigue life estimation [9].

Considering standard vibration testing with random signal with given frequency profile both assumptions for frequency domain methods are satisfied. However, when dealing with real service loads or environment conditions, the non-Gaussian and non-stationary signals are commonly observed. To what extent does the invalidity of those assumptions result on fatigue estimation has been studied from different aspects in classical $[10,11]$ and vibration fatigue. In latter a nonGaussian and non-stationary excitation signal is mechanically filtered by dynamic structure. An influence of non-Gaussian excitation to response signal and fatigue life was numerically investigated by Braccesi et al. [12], Rizzi et al. [13] and Kihm et al. [14], who used kurtosis and skewness as estimators of non-Gaussianity. NonGaussianity was also studied for vibration fatigue of composite structures [15] and MIMO test systems [16].

This manuscript presents an experimental and numerical analysis of non-Gaussianity and nonstationarity on vibration fatigue. Firstly, a dynamic structure of Y-shaped specimen [17] was experimentally excited with random stationary Gaussian signal to obtain fatigue parameters of used aluminium alloy. Secondly, a number of Y-specimens was excited with different sets of random signals, obtained by combining different kurtosis values and non-stationarities. As the non-

Corresponding author: janko.slavic@fs.uni-lj.si 
stationary non-Gaussian excitation was experimentally shown to be significantly more damaging compared to stationary non-Gaussian [18] the research later focused on experimental and numerical evaluation of nonstationarity rate of excitation signal. In this manuscript a run-test method [19] is proposed to obtain a nonstationarity index $\gamma$ [20]. Its applicability will be tested on a large number of fatigue tests and the relation between the amplitude-modulated non-stationarity and time-to-failure will be researched.

\section{Theoretical background}

In this chapter a condensed review of fundamental theory will be presented that is later applied to actual experimental data. Firstly, methods for identifying nonGaussianity and non-stationarity of the random signal will be shown. Secondly, general equations of motion for dynamic system are presented, which result in stress response PSD. Finally, a Tovo-Benasciutti frequency counting method is presented, which is used to obtain damage accumulation from stress response PSD.

\subsection{Random signal properties}

Given a discrete time-series of random process as $\mathbf{x}=\left[x_{1}, \ldots, x_{i}, \ldots, x_{n}\right]$, its $j$-th central moment $M_{j}$ and mean value $\mu$ are given as:

$M_{j}=\frac{1}{n} \sum_{i=1}^{n}\left(x_{i}-\mu\right)^{j}$,

$\mu=\frac{1}{n} \sum_{i=1}^{n} x_{i}$

and are based on Probability Density Function $P(x)$ (PDF). The PDF of Gaussian process is defined by mean value and second central moment (variance) of the process. For PDF definition of non-Gaussian processes a higher number of parameters is needed, introducing kurtosis $k_{u}$ as a measure of non-Gaussianity and defined as:

$$
k_{u}=M_{3} / M_{2}^{3 / 2} \text {, }
$$

which characterizes the sharpness of the PDF peak and the width of the PDF tails. By definition, Gaussian process has kurtosis of 3 . Non-Gaussian processes with high peak excursions have kurtosis higher than 3 .

On the other hand, quantification of non-stationarity in signal is not that straightforward, due to nature of nonstationarity itself. However, certain methods exist that monitor the changes in signal's statistical properties, which are for stationary process constant. Run-test [8] is a non-parametric method that divides a signal in time windows and calculates a variation of a chosen statistical variable over the whole signal. For each time window a chosen variable is met with a criterion related to whole signal; in presented study a condition was defined as:

$V(n)=\left\{\begin{array}{ll}1 ; & \left|R_{W}(n)-R_{T}\right|>\sigma_{R} \\ 0 ; & \left|R_{W}(n)-R_{T}\right| \leq \sigma_{R}\end{array}\right.$,

where $R_{W}(n)$ is a RMS value of a $n$-th window, $R_{T}$ a RMS value of the whole signal and $\sigma_{R}$ is a standard deviation of all window RMSs, as illustrated on signal time history on Fig. 1. A run is a sequence of identical observations $V$, followed and preceeded by different observation. Too many or too little runs in a whole signal is a proof of signal's non-stationarity. If probability of observation 1 or 0 is equal for each window, $N_{i}$ is a number of $i$ observations and $N$ is a total number of windows, then a mean value $\mu_{r}$ and variance $\sigma_{r}^{2}$ of runs is:

$\mu_{r}=\frac{2 N_{0} N_{1}}{N}+1, \quad \sigma_{r}^{2}=\frac{2 N_{0} N_{1}\left(2 N_{0} N_{1}-N\right)}{N^{2}(N-1)}$.

Any difference of actual number of runs $r$ from expected mean indicates a non-stationarity in signal. If a number of runs lays outside $\mu_{r} \pm \sigma_{r}$, a signal is considered nonstationary. Additionally, a non-stationarity index $\gamma$ is defined as:

$\gamma=r / \mu_{r} \quad[\%]$.

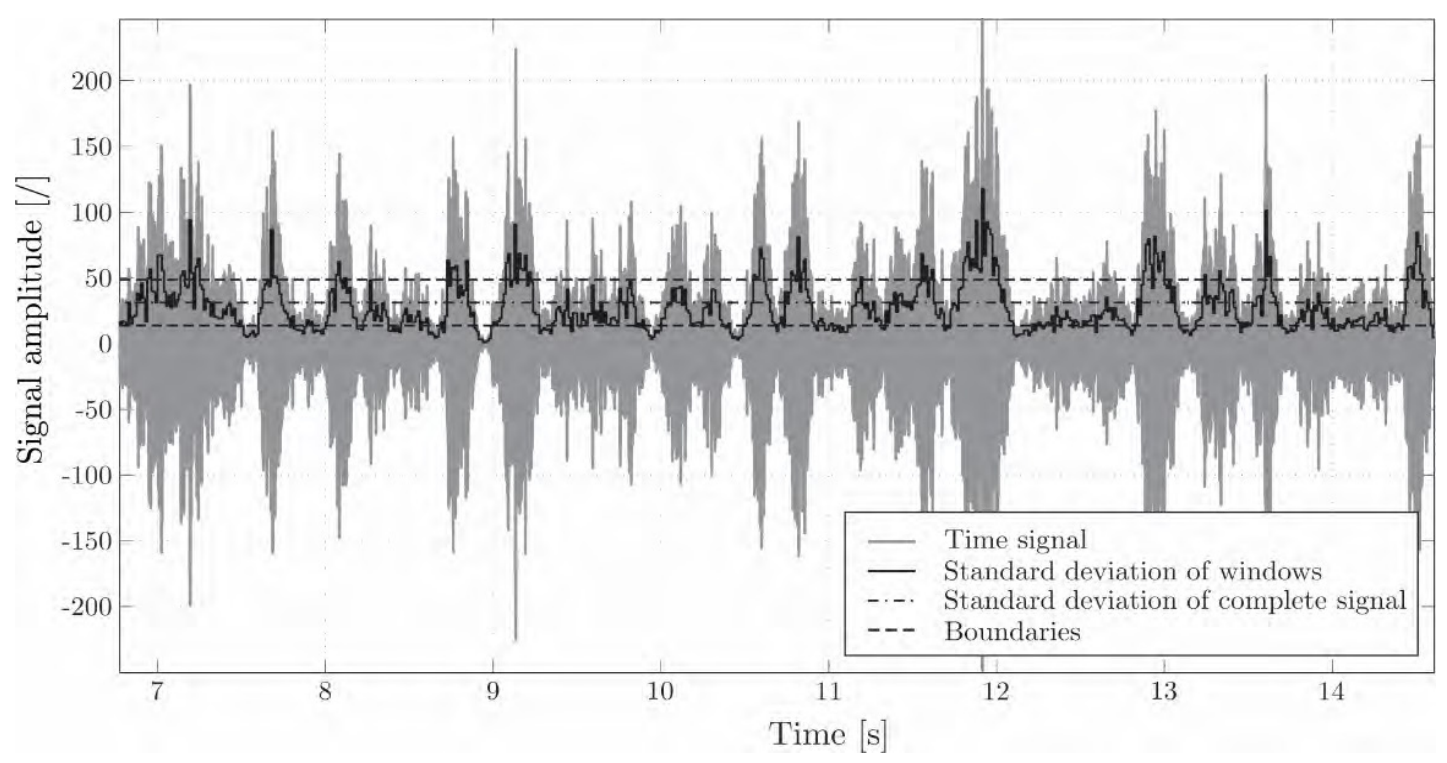

Fig. 1. Run-test evaluation of a non-stationary time signal. 


\subsection{Structural dynamics}

Flexible multi-degree-of-freedom structures respond to force excitation by respecting the equation of motion [21]:

$\mathbf{M} \ddot{\mathbf{x}}(t)+\mathbf{D} \dot{\mathbf{x}}(t)+\mathbf{K} \mathbf{x}(\mathrm{t})=\mathbf{F}(\mathrm{t})$.

In Eq. (7) $\mathbf{M}, \mathbf{D}$ and $\mathbf{K}$ present mass, damping and stiffness matrices, respectively; $\mathbf{x}$ and $\mathbf{F}$ are displacement and excitation force vectors. This coupled system of differential equations can be decoupled into

$\mathbf{I} \ddot{\mathbf{q}}(t)+\mathbf{I}\left[\mathbf{2} \xi \boldsymbol{\omega}_{\mathbf{0}}\right] \dot{\mathbf{q}}(t)+\mathbf{I}\left[\boldsymbol{\omega}_{0}^{2}\right] \mathbf{q}(\mathrm{t})=\boldsymbol{\Phi}^{\mathbf{T}} \mathbf{F}(\mathrm{t})$,

by introducing modal coordinates $\mathbf{x}=\mathbf{\Phi q}$, where $\boldsymbol{\Phi}$ is a modal matrix, consisting of mode shapes. $\boldsymbol{\omega}_{\mathbf{0}}$ and $\boldsymbol{\xi}$ are natural frequency and damping ratio vectors, respectively. By solving eigenvalue problem to obtain $r$ natural frequencies and modeshapes, uncoupled system of equations yields frequency response function on $j^{\text {th }}$ position for excitation on $k^{\text {th }}$ position:

$H_{j k}(\omega)=\sum_{r=1}^{n} \frac{\phi_{j r} \phi_{k r}}{\omega_{r}^{2}-\omega^{2}+2 \mathrm{i} \xi_{r} \omega_{r} \omega}$.

Combining response functions into response function matrix and knowing stress states of mode shapes, one can obtain stress response power spectral density (PSD) for given excitation PSD $\boldsymbol{S}_{\mathrm{ff}}(\omega)$ as:

$\mathbf{S}_{\boldsymbol{\sigma} \boldsymbol{\sigma}}(\omega)=\mathbf{H}_{\boldsymbol{\sigma} \mathbf{f}}^{*}(\omega) \mathbf{S}_{\mathrm{ff}}(\omega) \mathbf{H}_{\boldsymbol{\sigma f}}^{\mathrm{T}}(\omega)$.

\subsection{Damage accumulation}

Continuing from stress response PSD $\mathbf{S}_{\sigma \sigma}(\omega)$ an equivalent stress PSD $\boldsymbol{S}_{\text {eq }}(\omega)$ can be quickly obtained [22]. To employ frequency methods for fatigue calculation, $m$-th spectral moment should be defined [7]: $\lambda_{m}=\int_{0}^{\infty} \omega^{m} S_{e q}(\omega) \mathrm{d} \omega$.

Depending on the frequency method, a damage accumulation is a function of a list of spectral moments and material fatigue parameters $C$ and $b$, as included in Basquin's equation $\sigma=C N^{-1 / b}$ [23]. In this research, a Tovo-Benasciutti method [4] is proposed, that obtains damage accumulation $D$ using following equation

$D=\left(B_{T B}+\left(1-B_{T B}\right) \alpha_{2}^{B_{T B}-1}\right) \alpha_{2} D_{N B}$,

where $B_{T B}$ is a factor obtained from spectral moments $\lambda_{0}, \lambda_{1}, \lambda_{2}$ and $\lambda_{4} . D_{N B}$ is a damage for narrow band stress signal and depends on spectral moments $\left(\lambda_{0}, \lambda_{1}\right.$, $\left.\lambda_{2}, \lambda_{4}\right)$ and material fatigue parameters $(b, C)[3,4]$.

\section{Non-Gaussianity vs. non-stationarity}

The first part of the study focuses on non-Gaussianity and non-stationarity of random excitation signal and its influence on actual vibration fatigue life. For this purpose, a Y-shaped specimen is used, Fig. 2. Its geometry consists of three beams that are arranged at $120^{\circ}$ angles around the main axis and have a rectangular cross-section of $10 \times 10 \mathrm{~mm}$. The Y-shaped specimens

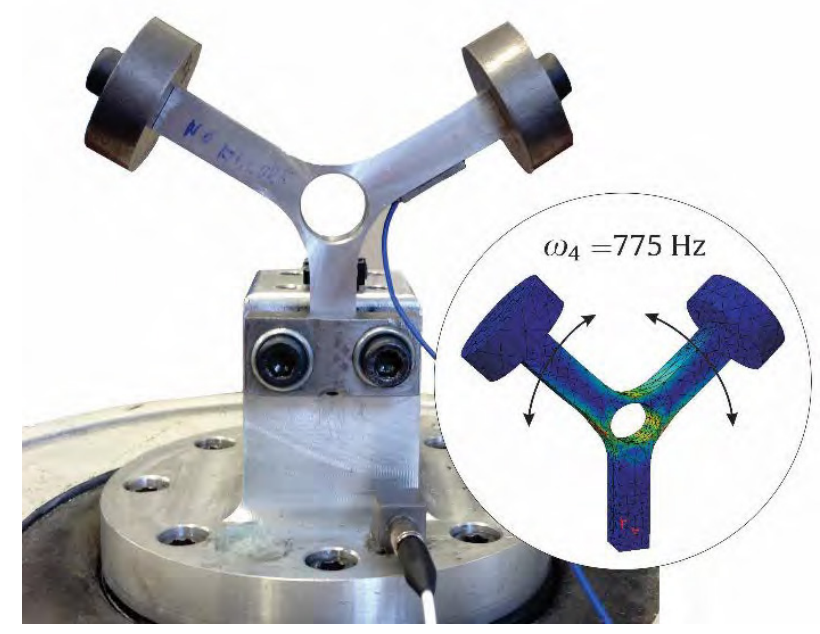

Fig. 2. Fixed Y-specimen with excited modeshape.

were made from the aluminium alloy A-S8U3; additional steel dead-weights with a mass of $52.5 \mathrm{~g}$ were added for natural-frequency tuning. The specimen was attached to electro-dynamic shaker and excited with flatshaped force PSD profile (Fig. 3) with frequency range $600-850 \mathrm{~Hz}$ so that specimen's $4^{\text {th }}$ natural frequency of $775 \mathrm{~Hz}$ lied within excited frequency band.

The procedure for evaluating non-Gaussianity and nonstationarity is following: first a set of specimens is excited with Gaussian stationary signals with flat PSD profiles at different amplitude levels (Fig. 3). By minimizing error between experimental and numerical fatigue lives material fatigue parameters $C$ and $b$ are obtained. Secondly, random signals with similar PSD profiles but different kurtosis and non-stationarities (Tab. 1) are generated and applied to the Y-specimens. Influence of non-Gaussianity and non-stationarity is then studied by comparing actual and predicted fatigue lives for given excitation signal type.

Tab. 1. Excitation signal types

\begin{tabular}{clc}
\hline Nr. & Signal type & $k_{u}$ \\
\hline 1. & Gaussian stationary & 2.96 \\
2. & Non-Gaussian stationary & 7.36 \\
3. & Non-Gaussian stationary & 5.43 \\
4. & Non-Gaussian non-stationary & 7.08 \\
\hline
\end{tabular}

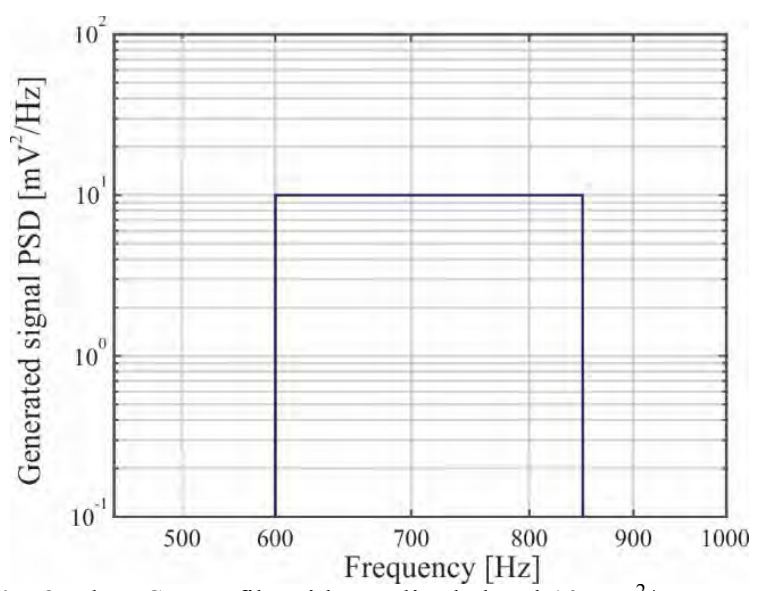

Fig. 3. Flat PSD profile with amplitude level $10 \mathrm{mV}^{2} / \mathrm{Hz}$. 
Four Y-shaped specimens were excited with random stationary Gaussian signal until fatigue failure occurred. The obtained fatigue lives are presented in Fig. 4. In order to obtain fatigue material parameters a numeric model was built and validated (Fig. 5). A function for numerical minimization of numerical error was proposed as:

$$
\begin{aligned}
\Delta T(b, C)=\sum_{i} & \left(\log _{10} T_{\text {act }, i}\right. \\
& \left.-\log _{10} T_{\text {est }, i}(b, C)\right)^{2},
\end{aligned}
$$

where $i$ denotes a single test specimen, $T_{\text {act }}$ is the measured fatigue life and $T_{\text {est }}$ is the estimated fatigue life of the specimen based on the numerical model. The minimization method showed strong convergence. Using the identified fatigue parameters, the Basquin's equation can be written as:

$$
\sigma=987.5 \cdot N^{-0.169} \text {. }
$$

Once fatigue parameters were known, a number of samples were excited with signals, where nonGaussianity and non-stationarity was combined according to Tab. 1. Time signals for signal types 3. and 4. with identical PSD profiles are presented in Figs. 6 and 7. The excitation force PSD level was precedently determined by measuring acceleration response of shaker's armature without Y-specimen [18]. Nonstationary signal was generated using amplitude modulation of stationary signal with beta-distribution signal $[18,20]$.

A total of nine Y-shaped specimens were tested under non-Gaussian excitation, three for each signal type 2-4. In order to evaluate the influence of the kurtosis on the fatigue life of tested specimens, the fatigue parameters

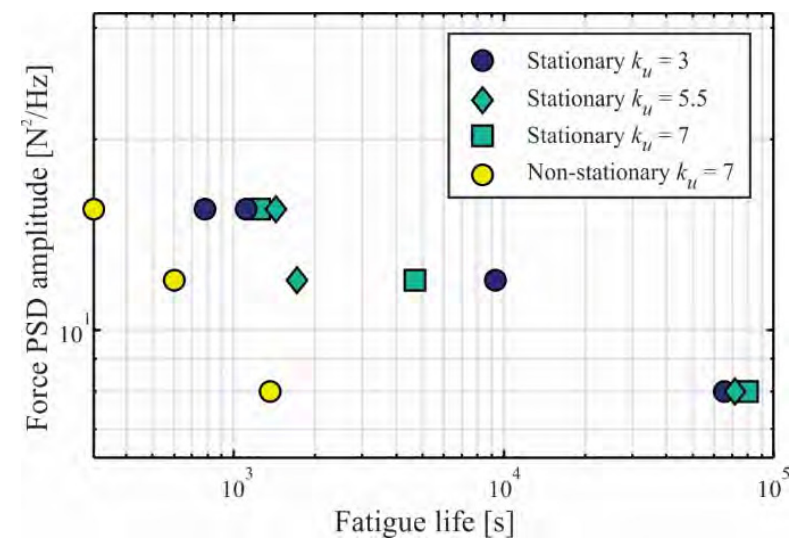

Fig. 4. Experimental fatigue lives.

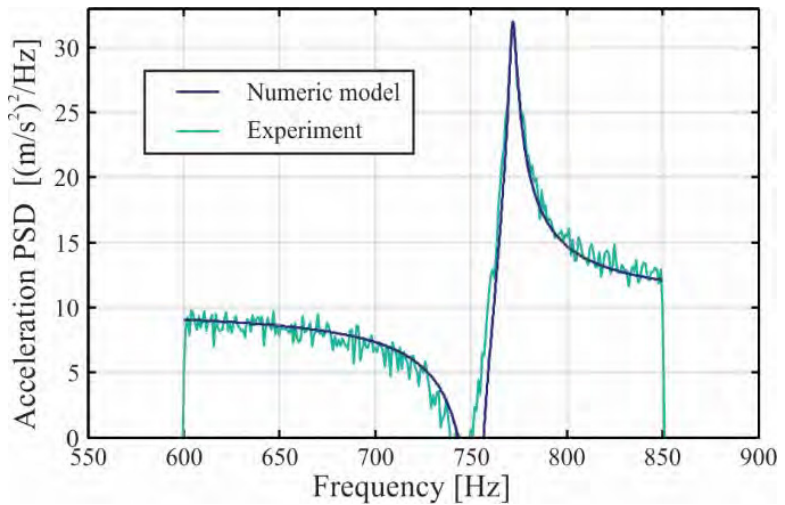

Fig. 5. Numerically and experimentally obtained response acceleration PSD. obtained under the Gaussian condition are also used for a numerical estimation of the fatigue life for the case of non-Gaussian excitation. In order to investigate when it is justified to apply Gaussian-based counting methods to

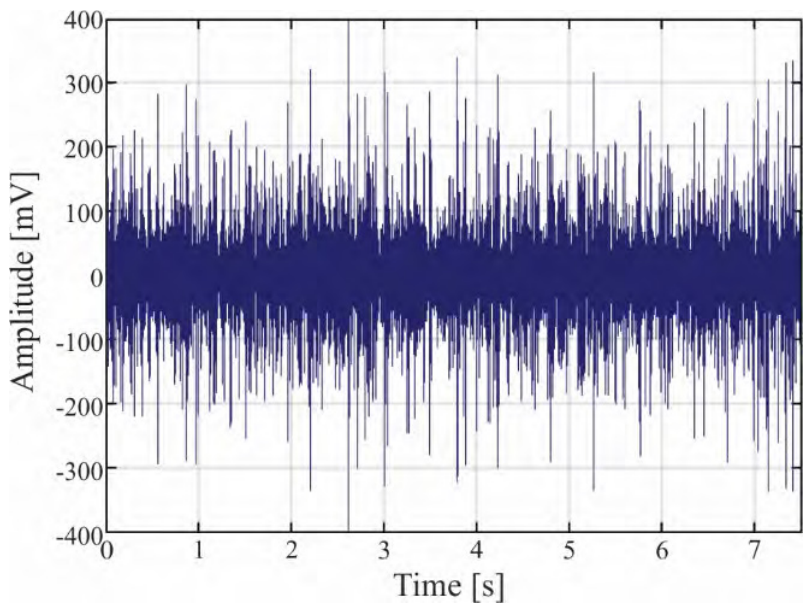

Fig. 6. Stationary non-Gaussian signal with $\mathrm{k}_{\mathrm{u}}=7$.

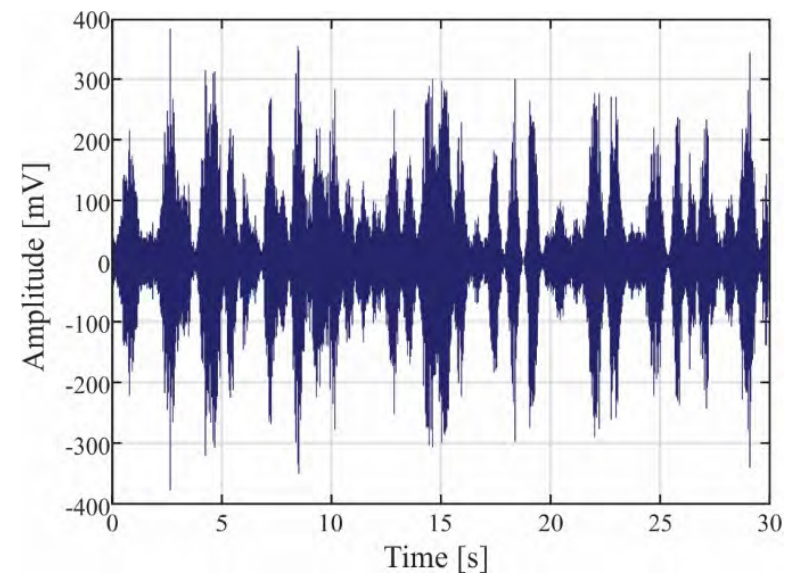

Fig. 7. Non-stationary non-Gaussian signal with $k_{u}=7$.

non-stationary excitation, the Tovo-Benasciutti counting method was also used in the case of the non-Gaussian excitations.

A comparison between the experimental and calculated fatigue lives is shown in Fig. 8. From Fig. 8 it is noticeable that the stationary, non-Gaussian excitation differs only slightly if compared to the calculated fatigue life. In contrast to this case, for the case of nonstationary non-Gaussian excitation, the comparison shows how the difference between the calculated fatigue lives and the actual fatigue lives is significant. From this comparison one can conclude that for the case of stationary excitation the use of standard frequencycounting methods [9] available in the literature gives reliable results comparable to reality, while in the case of strongly non-stationary signals the use of the same counting methods supplies uncorrected results. Also, Fig. 4 shows the relation between the excitation force PSD amplitude and the experimental fatigue life; again, the non-stationary experiments clearly differentiate from the stationary experiments. 
The latter difference between the calculated and measured fatigue lives arises due to the non-Gaussianity of the stress response. For this reason, a piezo-electric strain gauge was applied on the specimen's beam, as shown in Fig. 2, to monitor the stress response. For the case of stationary non-Gaussian loadings the kurtosis of the stress is always around the value 3 , confirming that due to the stationarity of the excitation, the response of the structure is Gaussian. However, for the case of nonstationary non-Gaussian loadings the output kurtosis is significantly higher, although it is still lower than the kurtosis of the input signal. In any case, we can conclude that if the input signal is quasi-stationary, the output kurtosis always tends to the Gaussianity; however, if the input signal is non-stationary, the stress response remains strongly non-Gaussian and leads to shorter fatigue lives than expected.

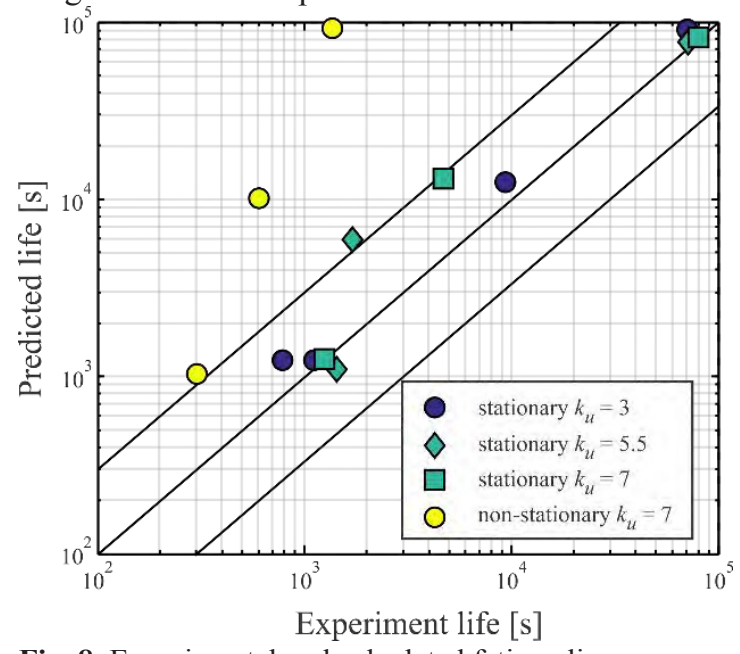

Fig. 8. Experimental and calculated fatigue lives.

\section{Non-stationarity index}

The following section focuses on quantification of nonstationarities in excitation signal and on identification of non-stationary signals that result in reduced vibration fatigue life.

\subsection{Signal generation}

In order to investigate this first a set of time signals with different non-stationarity rates was obtained. For the bulk of physical phenomena the non-stationarity is exhibited as the time variance of a signal's power; the fluctuations in signal's frequency content are less commonly observed.

Therefore, beta-distribution amplitude modulation of a stationary signal with flat-shaped PSD profile was initially performed with a carrier wave, as proposed in Sec. 3 (Fig. 7) and described in detail in [14] and [18].

In the next step the initial carrier wave with time duration of 30 minutes was then squeezed by a factor of 2 and again multiplied with flat-profiled stationary signal

. This signal was denoted as SQ-2, whereas the amplitude modulated signal with original (non-squeezed carrier wave) was denoted as SQ-1. In a similar manner, additional 5 nonstationary signals were generated for different squeezing factors 4, 10, 50, 500 and 10000. After squeezing, each signal was repeated to reach the

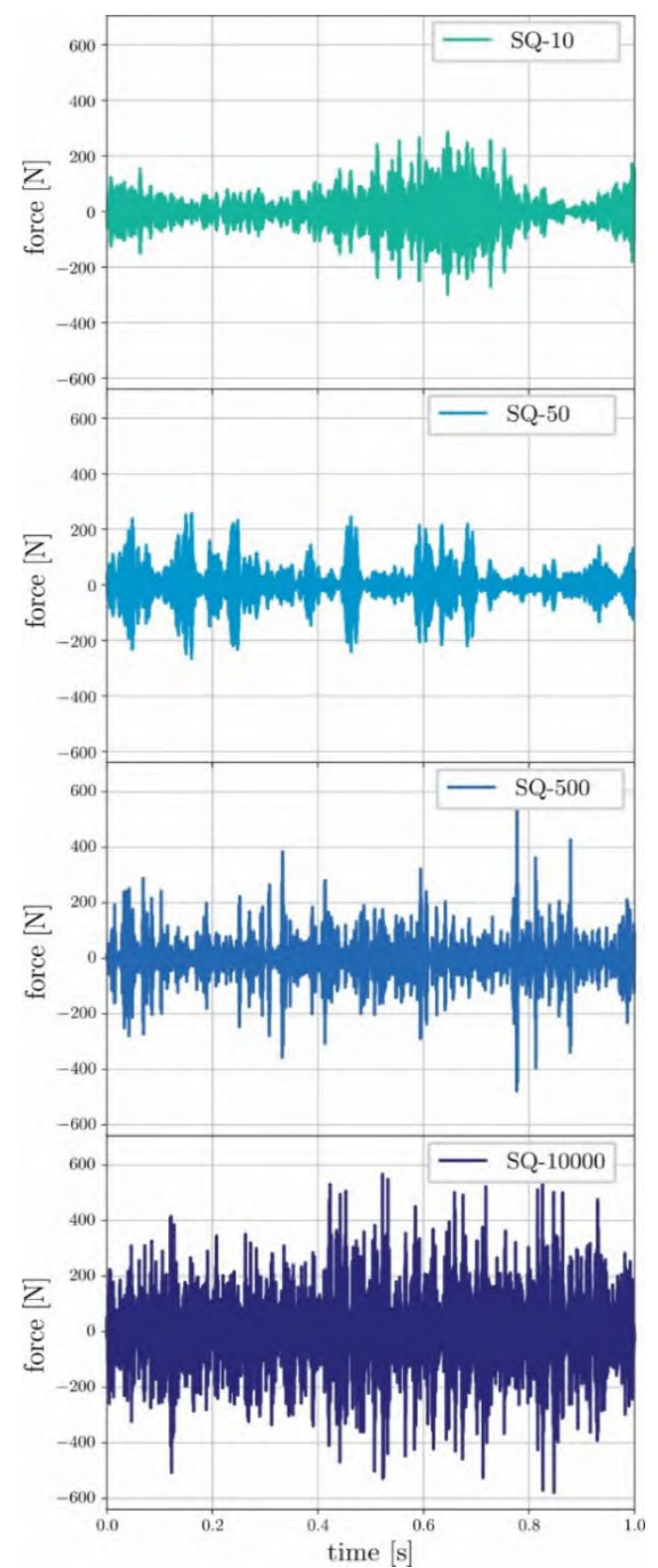

Fig. 9. Time history of measured excitation signals SQ-10, SQ-50, SQ-500 and SQ-10000 with PSD level of $13.5 \mathrm{~N}^{2} / \mathrm{Hz}$.

time length of 30 minutes. All time signals had the same PSD, the same time length, the same kurtosis, but different levels of non-stationarity. Squeezed signals SQ10, SQ-50, SQ-500 and SQ-10000, that were measured on electro-dynamic shaker are presented in Fig. 9.

\subsection{Signal evaluation with run-test method}

Here, the signals later used for $\mathrm{Y}$-specimen excitation and with PSD level of $13.5 \mathrm{~N}^{2} / \mathrm{Hz}$ are evaluated with two-sided run-test method, presented in Sec. 2.1 and Fig. 1. As known [3] run-test method is sensitive to the width of the windows, to which the signal is divided. Consequently, all 8 signals (7 non-stationary and 1 de facto stationary) were evaluated for different time lengths of dividing windows. In total seven time-window 
widths were applied to the run-test, ranging from 0.005 to $1 \mathrm{~s}$. In Tab. 2 the resulting non-stationarity indexes $\gamma$ are given. Moreover, the influence of the window's width is graphically presented in Fig. 10. According to Rizzi et al. [13] study the optimal window width is expected to be related to the period of

Tab. 2. Non-stationarity index $\gamma$ for two-sided method: results in bold identify signal as stationary.

\begin{tabular}{cc|c|c|c|ccc}
\hline \multirow{2}{*}{$\begin{array}{c}\text { Signal } \\
\text { type }\end{array}$} & \multicolumn{7}{c}{ Window width } \\
\cline { 2 - 7 } & $0.005 \mathrm{~s}$ & $0.01 \mathrm{~s}$ & $0.0125 \mathrm{~s}$ & $0.02 \mathrm{~s}$ & $0.04 \mathrm{~s}$ & $0.1 \mathrm{~s}$ & $1 \mathrm{~s}$ \\
\cline { 2 - 7 } & \multicolumn{7}{c}{ Non-stationarity index $\gamma[\%]$} \\
\hline Stationary & 96 & $\mathbf{9 9}$ & $\mathbf{1 0 0}$ & $\mathbf{1 0 1}$ & $\mathbf{1 0 2}$ & $\mathbf{9 9}$ & 72 \\
SQ-10000 & 96 & $\mathbf{1 0 1}$ & $\mathbf{1 0 1}$ & $\mathbf{1 0 1}$ & $\mathbf{1 0 2}$ & $\mathbf{1 0 0}$ & $\mathbf{1 0 1}$ \\
SQ-500 & 95 & 97 & $\mathbf{9 9}$ & $\mathbf{9 9}$ & $\mathbf{1 0 2}$ & 105 & $\mathbf{9 0}$ \\
SQ-50 & 73 & 86 & 92 & 98 & 95 & 96 & 114 \\
SQ-10 & 65 & 62 & 61 & 61 & 72 & $\mathbf{9 6}$ & $\mathbf{1 0 9}$ \\
SQ-4 & 64 & 60 & 57 & 50 & 46 & 67 & $\mathbf{1 0 5}$ \\
SQ-2 & 65 & 60 & 58 & 50 & 40 & 39 & $\mathbf{1 0 4}$ \\
SQ-1 & 66 & 62 & 60 & 53 & 40 & 31 & $\mathbf{9 6}$ \\
\hline
\end{tabular}

\begin{tabular}{|l|l|l|l|l|l}
\hline & [14]; in the case of the Y-specimen the period of the \\
impulse response was experimentally determined to be \\
in the range up to $0.2 \mathrm{~s}$. By inspecting Fig. 10 a \\
significant difference between low squeezing and high \\
squeezing is observed; at a window width of $0.04 \mathrm{~s}$ the \\
signals SQ-1 to SQ-4 were identified as non-stationary, \\
while the SQ-500, SQ-10000 and also de facto stationary \\
signal were identified as stationary. Similar results were \\
found for other RMS values of the excitation signal.
\end{tabular}

\subsection{Vibration fatigue testing}

Here, the de facto stationary and squeezed signals SQ-1 SQ-10000 were applied to the Y-specimen. According to a preliminary analysis of the excitation signals the nonstationarity index $\gamma$ shows that the signals SQ-500, SQ10000 can be considered as stationary and the signals SQ-1, SQ-2 and SQ-4 can be considered as nonstationary. This finding will now be tested against the actual vibration fatigue life.

For the sake of experimental comprehensiveness the time signals were applied to the Y-specimen at four different force PSD levels, as shown in Fig. 11. For each of the 19 combination pairs of excitation PSD level and signal type, two samples were teste. In total, 41 samples were broken. Certain load level and signal type combinations were left untested either due to instantaneous failure or due to the absence of failure after $2 \times 10^{7}$ load cycles.

The fatigue life for all tested specimens is given in Tab. 3 . It is clear that squeezing the carrier wave and thus

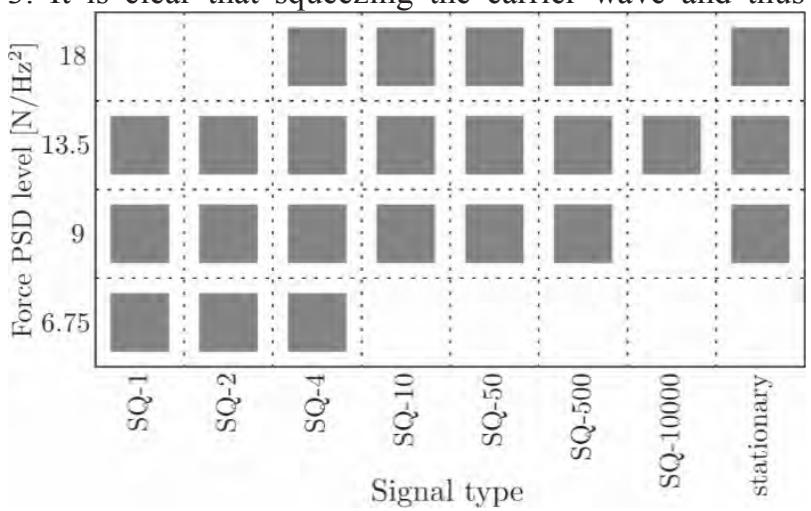

the system's impulse response, i.e., the time in which the response amplitude reduces to $10 \%$ of the initial value

Fig. 11. Experimentally tested combinations of signal types and excitation levels. 
changing the non-stationarity rate significantly changes the fatigue life, see Fig. 12. From Fig. 12 it is clear that Tab. 3. Experimental fatigue lives [s] of tested Y-specimens. for the SQ-4 the fatigue life is significantly shorter than

\begin{tabular}{lllllllll}
\hline Force PSD & \multicolumn{7}{c}{ Signal type } \\
\cline { 2 - 9 } level $\left[\mathrm{N}^{2} / \mathrm{Hz}\right]$ & SQ-1 & SQ-2 & SQ-4 & SQ-10 & SQ-50 & SQ-500 & SQ-10000 & Stationary \\
\hline \multirow{2}{*}{18} & $/$ & $/$ & 643 & 598 & 999 & 1624 & $/$ & 3157 \\
& & & 341 & 497 & 1069 & 2793 & 6299 & 4428 \\
13.5 & 961 & 871 & 737 & 1175 & 1120 & 4069 & 6309 & \\
& 857 & 907 & 899 & 1333 & 5234 & 6411 & $>12600$ \\
9 & 2364 & 2853 & 3279 & 3177 & 3722 & $>12600$ & $/$ & \\
6.75 & 2994 & 2391 & 2899 & 3824 & 5894 & & $/$ & $/$ \\
\hline
\end{tabular}

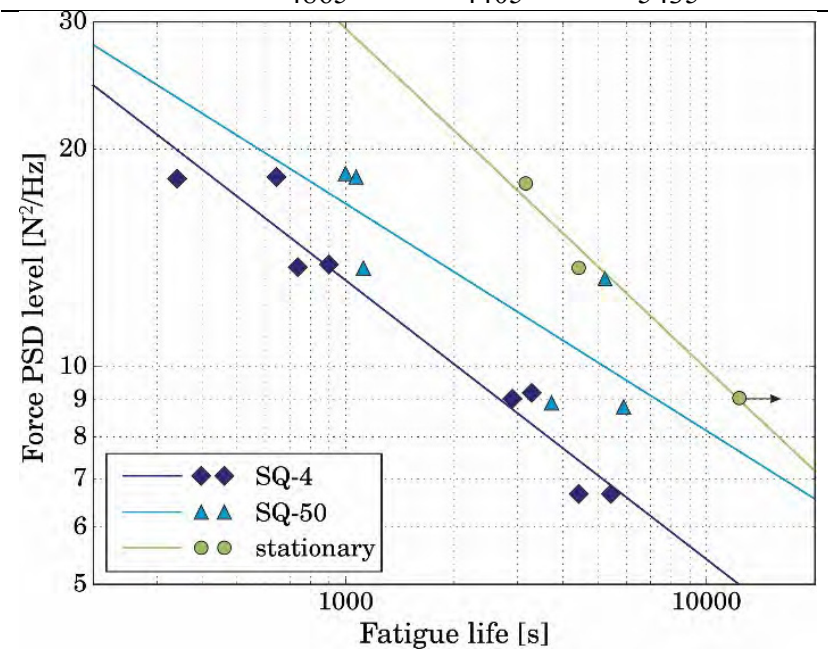

Fig. 12. Experimental fatigue life for the representative excitation signal types: SQ-4, SQ-50 and de facto stationary.

for the de facto stationary signal, while SQ-50 is in between.

Due to the considerable influence of the non-stationarity rate on the fatigue life only tests with an excitation force PSD level of $13.5 \mathrm{~N}^{2} / \mathrm{Hz}$ were possible on the complete set of generated non-stationary signals SQ-1 - SQ-10000 and on the de facto stationary signal, see Tab. 3. To better understand Fig. 12, a detailed analysis of the PSD level of $13.5 \mathrm{~N}^{2} / \mathrm{Hz}$ is shown in Fig. 13: one axis shows the fatigue life, the other axis shows the non-stationarity index. It was previously shown that the excitation signals from SQ-1 - SQ-4 can be considered as non-stationary, while signals SQ-500 and above can be considered as stationary. As all the time signals had the same PSD, the same time length, the same kurtosis, but different rates of non-stationarity one can conclude that of nonstationarity results in significant differences in fatigue life. The difference between non-stationary and stationary conditions is approximately 5 -fold.

Fig. 14 shows the fatigue life normed to the fatigue life during stationary excitation. From the results of all 41 tested samples it is clear that the excitation identified as non-stationary resulted in a reduced fatigue life to approximately $20 \%$. Signals that are not clearly stationary, nor are they non-stationary, have a fatigue life between the two groups. Based on presented results it is reasonable to conclude that non-stationary index $\gamma$ provides reliable results to distinguish between nonstationary excitation signals that do or do not reduce vibration fatigue life when compared to stationary excitation.

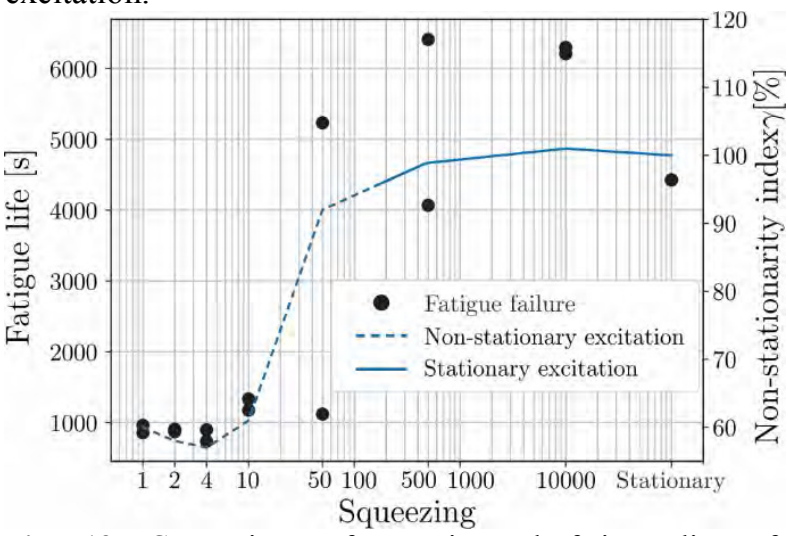

Fig. 13. Comparison of experimental fatigue lives for excitation PSD level of $13.5 \mathrm{~N}^{2} / \mathrm{Hz}$ and excitation signal's nonstationarity index $\gamma$ for window width of 0.0125 seconds using two-sided run-test method.

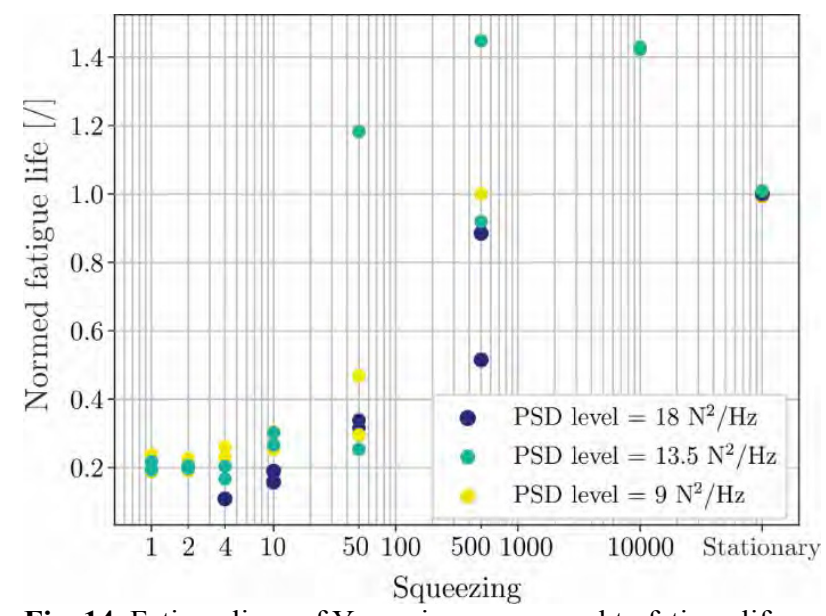

Fig. 14. Fatigue lives of $Y$-specimens, normed to fatigue life under de facto stationary excitation signal.

\section{Conclusions}

In the presented research the fatigue life of a simple Yshaped specimen was investigated in order to determine how a change in the excitation kurtosis and stationarity affects the fatigue life of a real structure. Firstly, several experimental verifications of random excitation signals with the same PSDs, but with different kurtosis were performed. In the case of stationary random excitations, it was found that non-Gaussian signals with a stationary rate produced a Gaussian response, while in the case of 
non-stationary random signals, the response of the structure is non-Gaussian. The obtained results show that the fatigue life due to amplitude-modulated nonstationary excitations is significantly shorter when compared to the fatigue lives obtained under the valid condition of stationarity. Moreover, it was found that if the non-Gaussian excitation is stationary, the calculated fatigue lives with classical frequency-counting methods are comparable to the fatigue lives under Gaussian excitation; therefore, justifying the use of frequencycounting methods, even if validated only in the case of Gaussian excitations. In contrast, for the case of burst, non-Gaussian excitation, the obtained fatigue life exhibits a significantly higher damage accumulation compared to the fatigue lives attained under Gaussian signals. For this reason, considering a non-stationary non-Gaussian excitation as Gaussian and, consequently adopting the classic frequency-counting methods may result in a wrong fatigue-life estimation.

To this end, a study later focused on identifying critical non-stationary amplitude-modulated in terms of actual fatigue life. Excitation is frequently non-stationary in terms of time-varying power and the question is what rate of non-stationarity can still be considered as stationary and how does the rate of non-stationarity effect the fatigue life? To answer this, additional experimental tests of $\mathrm{Y}$-specimens were performed using squeezed signals with the same PSD and kurtosis, but different rates of non-stationarity. An enhanced method to identify the non-stationarity was proposed and resulted in a clear differentiation of the non-stationarity in the signal.

The signals that were identified as non-stationary resulted in a significantly shorter fatigue life of the sample than the ones that were identified as (or were de facto) stationary. Finally, the non-stationarity identification relates on the natural frequencies of the researched structure. However, if the non-stationarity is identified during the excitation, the resulting fatigue life was shown to significantly decrease (in this research to $1 / 5$ th).

1. United States Department of Defense, MIL-STD$810 F$, Department of defense test method standard for environmental engineering consideration and laboratory tests (2000)

2. N. W. M. Bishop, The use of frequency domain parameters to predict structural fatigue, University of Warwick (1988)

3. T. Dirlik, Application of computers in fatigue analysis, University of Warwick (1985)

4. D. Benasciutti, R. Tovo, Spectral methods for lifetime prediction under wide-band stationary random process, Int. J. Fatigue, 27(8): pp. 867-877 (2005)

5. C. Braccesi, F. Cianetti, L. Tomassini, Fast evaluation of stress state spectral moments, Int. J. of Mech. Sci., 127: pp.4-9 (2017)

6. M. Mršnik, J. Slavič, M. Boltežar, Multiaxial vibration fatigue - a theoretical and experimental comparison, Mech. Sys. Signal Proc., 76-77: pp. 409-423 (2016)

7. M. Mršnik, J. Slavič, M. Boltežar, Vibration fatigue using modal decomposition, Mech. Sys. Signal Proc., 98: pp. 548-556 (2018)

8. S. Bendat, G. Piersol, Random data: analysis and measurement procedures, $4^{\text {th }}$ edition, Wiley\&Sons (2010)

9. M. Mršnik, J. Slavič, M. Boltežar, Frequencydomain methods for a vibration-fatigue-life estimation - application to real data, Int. J. Fatigue, 47: pp. 8-17 (2013)

10. D. Benasciutti, Fatigue analysis of random loadings, University of Ferrara (2004)

11. A. Niesłony, M. Bohm, T. Łagoda, F. Cianetti, The use of spectral method for fatigue life assessment for non-Gaussian random loads, Acta Mech. Automat., 10(2): pp. 100-103 (2016)

12. C. Bracessi, F. Cianetti, G. Lori, D. Pioli, The frequency domain approach in virtual fatigue estimation of non-linear system: the problem of nonGaussianity state of stress, Int. J. Fatigue, 31(4): pp. 766-775 (2009)

13. S. A. Rizzi, A. Prezekop, T. Turner, On the response of a non-linear structure to high-kurtosis nonGaussian random loadings, in: $8^{\text {th }}$ Eurodyn, Leuven (2011)

14. F. Kihm, S. A. Rizzi, N. S. Fergusson, A. Halfpenny, Understanding how kurtosis is transferred from input acceleration to stress response and its influence on fatigue life, in: $11^{\text {th }}$ RASD, Pisa (2013)

15. Z. Fan, Y. Jiang, S. Zhang, J. Tao, X. Chen, Research on vibration fatigue of carbon fibrereinforced composites under non-Gaussian random load, In: PHM-2017, Harbin (2017)

16. R. Zheng, H. Chen, X. He, W. Zheng, Probability distributions control for multi-input multi-output stationary non-Gaussian random vibration test, J. Vib. Control, DOI: 10.1177/1077546317747503 (2017)

17. M. Česnik, J. Slavič, M. Boltežar, Uninterrupted and accelerated vibrational fatigue testing with simultaneous monitoring of the natural frequency and damping, 331(24): pp. 5370-5382 (2012)

18. M. Palmieri, M. Česnik, J. Slavič, F. Cianetti, M. Boltežar, Non-Gaussianity and non-stationarity in vibration fatigue, Int. J. Fatigue, 97: pp. 9-19 (2017)

19. V. Rouillard, Quantifying the non-stationarity of vehicle vibrations with the run test, Packag. Technol. Sci., 27(3): pp. 203-219 (2014)

20. L. Capponi, M. Česnik, J. Slavič, F. Cianetti, M. Boltežar, Non-stationarity index in vibration fatigue: Theoretical and experimental research, Int. J. Fatigue, 104: pp. 221-230 (2017)

21. N. M. M. Maia, J. M. M. Silva, Theoretical and experimental modal analysis, $1^{\text {st }}$ edition, Research Studies Press (1997) 
22. X. Pitoiset, A. Preumont, Spectral methods for multi-axial random fatigue analysis of metallic structure, Int. J. Fatigue, 22(7): pp. 541-550 (2000)

23. Y.-L. Lee, J. Pan, R. Hathaway, M. Barkey, Fatigue testing and analysis: Theory and practice, $1^{\text {st }}$ edition, Elsevier (2004) 